\title{
Low-dose cranial boost in high-risk adult acute lymphoblastic leukemia patients undergoing bone marrow transplant
}

\author{
William Su, BA ${ }^{\mathrm{a}}$, Marcher Thompson, $\mathbf{M D}^{\mathrm{b}}$, Ren-Dih Sheu, $\mathbf{P h D}^{\mathrm{b}}$, Amir Steinberg, $\mathbf{M D}^{\mathrm{c}}$, Luis \\ Isola, $\mathbf{M D}^{\mathrm{c}}$, Richard Stock, $\mathbf{M D}^{\mathrm{b}}$, and Richard L. Bakst, $\mathbf{M D}^{\mathrm{b}}{ }^{,}$ \\ alcahn School of Medicine at Mount Sinai, New York, New York

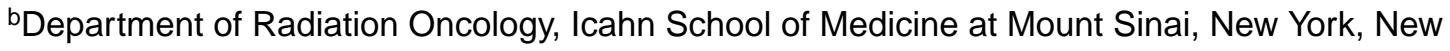 \\ York \\ 'Department of Medical Oncology, Icahn School of Medicine at Mount Sinai, New York, New York
}

\begin{abstract}
Purpose-Acute lymphoblastic leukemia (ALL) has a predilection for CNS involvement. Patients with high-risk ALL are often managed with transplant using a radiation-based conditioning regimen. Historically, a high-dose prophylactic cranial boost (CB) of $\geq 12$ Gy was given to reduce risk of central nervous system (CNS) recurrence. However, the use of CB has fallen out of favor because of toxicity concerns. In high-risk adults undergoing transplant at our institution, we have used a low-dose $6 \mathrm{~Gy} \mathrm{CB}$ to reduce toxicity while conditioning adults with fully developed brains. The safety, efficacy, and utility of a low-dose CB in adults are poorly studied; herein, we report their outcomes and toxicity.
\end{abstract}

Methods and materials-We identified all high-risk ALL patients undergoing total body irradiation as part of their conditioning regimen. Those who received $6 \mathrm{~Gy} \mathrm{CB}$ or no $\mathrm{CB}$ were included (55 total). Their charts were reviewed and statistical analyses were completed with $\mathrm{R}$, version 2.15.2.

Results-In patients undergoing CB, 3-year CNS disease-free survival and overall survival were $94.7 \%$ and $62.7 \%$. In those not undergoing CBs, survivals were $81.8 \%$ and $51.5 \%$. Notably, within the $\mathrm{CB}$ cohort, patients without prior CNS involvement had no CNS failures. In contrast, in the non-CB cohort, there were 2 CNS failures in patients with no history of CNS involvement. In the $\mathrm{CB}$ cohort, the only notable acute toxicity was parotitis (2.8\%). Late toxicity in the CB cohort included 1 instance of cataracts (2.8\%) without any evidence of cognitive impairment or potential radiation induced secondary malignancy.

Conclusions-A dose of 6 Gy CB is well-tolerated in the adult ALL population as part of a radiation-based conditioning regimen. Low-dose CB may be considered in adult patients with high-risk ALL without prior CNS involvement to reduce the likelihood of recurrence.

*Corresponding author. Radiation Oncology Associates, 1184 Fifth Ave., 1st Floor, Box 1236, New York, NY 10029.

Richard.Bakst@mountsinai.org (R.L.Bakst).

Conflicts of interest: None. 


\section{Introduction}

High-risk acute lymphoblastic leukemia (ALL) patients often require stem cell transplant to manage their disease. Studies have shown that transplant increases survival in high-risk pediatric and adult patients. ${ }^{1}$ The National Cancer Institute defines high-risk patients as those who have failed induction therapy, relapse, genetic abnormalities such as the BCRABL transformation (Philadelphia chromosome), or are older than 13 years old. ALL is uncommon in adults and not well studied. Additionally, it can be difficult to treat adults because they do not respond as well to chemotherapy as children do. ${ }^{2-4}$ This is consistent with data that show that cure rates in adult ALL remain low compared with childhood ALL and rarely exceed $40 \%$, despite frequent use of transplant. ${ }^{1,2,5}$ Survival has a strong inverse relationship with age, and adult ALL patients require alternative treatment strategies. ${ }^{4}$

In high-risk ALL cases, patients often receive stem cell transplant to manage their disease. This requires a pretransplant myeloablative conditioning regimen typically consisting of radiation and chemotherapy such as total body irradiation (TBI) with cyclophosphamide before transplant. Additionally, ALL has a predilection for the central nervous system (CNS). Because of this, additional boosts of radiation are often given prophylactically to sanctuary sites such as the brain and testes. Before CNS prophylaxis was used on patients, up to $75 \%$ of pediatric recurrences of disease occurred in the CNS. ${ }^{2}$ A landmark study completed in 1980 by Omura et al established the importance of using CNS prophylaxis to reduce instances of CNS relapse in adult ALL patients. ${ }^{6}$ Because CNS recurrence confers poor prognosis, preventing it is integral to improving overall survival. Nowadays, there is controversy about the best way to administer CNS prophylaxis in a manner that would optimize its risks and benefits.

CNS prophylaxis can include cranial irradiation and or intrathecal chemotherapy. Typical dosages of $\geq 12$ Gy were used in a prophylactic cranial boost $(\mathrm{CB})$ in the past. ${ }^{7,8}$ However, these CBs have fallen out of favor because of associated cognitive side effects and toxicity, particularly in the treatment of children. There are many studies completed in children with these higher dosage CBs that suggest that high-dose prophylactic intrathecal chemotherapy is a viable alternative in preventing CNS recurrence. ${ }^{9,10}$ However, Waber et al also noted that it is possible that cranial radiation at lower dosages may not add significant neurotoxicity and may be considered in certain situations. ${ }^{9}$ It is important to note that the majority of CB toxicity data is derived from pediatric literature. This has discouraged general $\mathrm{CB}$ usage without thorough evaluation in adults regarding dosage and toxicity.

Some studies have suggested that intrathecal methotrexate (IT MTX), which has been commonly suggested as a viable alternative to $\mathrm{CB}$, can lead to focal cognitive defects and neurotoxicity. ${ }^{11,12}$ Conklin et al noted that omitting cranial radiation in children may help preserve cognitive abilities, but the chemotherapy used to replace $\mathrm{CB}$ also came with cognitive risks. In children with ALL who received higher intensity chemotherapy and no $\mathrm{CB}$, the majority performed below average in various cognitive tests. ${ }^{13}$ It is clear that both radiation and chemotherapy have side effects and their usage should be carefully considered depending on the patient's disease characteristics and tolerance for each form of treatment. 
Although a retrospective study has been done observing the efficacy of $6 \mathrm{~Gy} \mathrm{CB}$ compared with IT MTX in the past, it observed only children with ALL who received treatment. ${ }^{14}$

The majority of literature on ALL treatment investigates children because that is the population with the most common occurrence of the disease. Because of the rarity of adult ALL, the efficacy and toxicity of a lower 6 Gy CB in adults is not well documented. As a result, there is no standard regimen to administer cranial prophylaxis in adults. There is less concern for long-term cognitive effects of radiation in adults, so it is possible that CB may not be as toxic in adults compared with children. ${ }^{15}$

To address CB-associated toxicity at our institution, we have used a lower 6 Gy prophylactic $\mathrm{CB}$ before TBI in conditioning high-risk ALL adults for transplant for many years. Herein we conducted a retrospective study to observe the role of a lower dose 6 Gy prophylactic CB in the treatment of adults with high-risk ALL. We were interested in analyzing our experiences to document efficacy and toxicity so we could better understand CNS treatment in adults.

\section{Methods and materials}

Patient characteristics

After receiving approval from the institutional review board, ALL patients who underwent transplant at our institution were identified. Fifty-five total patients met inclusion criteria for high-risk disease and were treated between 2000 and 2015. Median age at transplant was 37 (range, 14-58) in the CB group and 35 in the non-CB group (range, 4-66) (unpaired $t$ test, $P$ $=.7013)$. $\mathrm{CB}$ was considered for patients ages 14 and older, given that the age cutoff meets the National Cancer Institute definition for high-risk ALL. The most prevalent form of leukemia that presented in patients was high-risk B-cell ALL (69.1\%). Further patient characteristics are included in Table 1.

\section{Conditioning regimen}

In the $\mathrm{CB}$ group, patients received a $6 \mathrm{~Gy} \mathrm{CB}$ as part of a $\mathrm{TBI}$ conditioning regimen for transplant. A 6 Gy $\mathrm{CB}$ was used in an effort to reduce treatment-related toxicity, while still achieving a cumulative 18 Gy dose to the brain when given along with 12 Gy TBI. Patients received $\mathrm{CB}$ in $2 \mathrm{~Gy}$ fractions over 3 days (6 Gy total) with the opposed laterals technique before TBI (median 12 Gy, range 12-15 Gy). For CB, the whole brain was covered along with the posterior third of the retina. Patients at our institution begin CB on Wednesday and receive 2 Gy over 3 days until Friday. They are admitted over the weekend and receive TBI twice daily over the course of 3 to 4 days starting on Monday and then receive transplant after TBI regimen is completed.

The TBI treatment is delivered by using a Varian 21iX LINAC 6MV photon beam with a midplane dose rate of approximately 8 to $13 \mathrm{cGy} / \mathrm{min}$. The patient is treated in a standing position resting against a stand specially designed for TBI. A field size of $38 \times 38 \mathrm{~cm}$ at 100 $\mathrm{cm}$ source to axis distance is used. The gantry angle is set at $275^{\circ}$. The collimator is angled $45^{\circ}$ to produce a diamond-shaped field with the patient being at its center. Machine dose rate is set to $300 \mathrm{mu} / \mathrm{min}$. A Lexan screen ( $1 \mathrm{~cm}$ thick) is placed between the patient to increase 
skin dose and customized lung blocks are used during the treatment. Patients are typically treated 2 times per day (anteroposteriorly then posteroanteriorly) with at least 6-hour intervals.

Additionally, a group of ALL transplant patients who did not receive CB but received TBI (median, $12 \mathrm{~Gy}$; range, $0.2-15 \mathrm{~Gy}$ ) was queried for comparative purposes. A total of $42.1 \%$ of these patients received single-fraction TBI. A total of $72.7 \%$ of patients had received intrathecal chemotherapy before transplant as a part of their induction regimens. Twelve of the 22 males in the total population received testes boost as well. Those with prior CNS disease had all received IT chemotherapy before transplant as part of their chemotherapy regimens. Overall, $72.2 \%$ of CB group were indicated for IT chemotherapy and $73.7 \%$ of the TBI only group received IT chemotherapy. The primary form of chemotherapy given IT was methotrexate.

\section{Follow-up and data analysis}

The follow-up time was calculated from the date of transplant. Posttransplant outcomes including disease status, toxicity, graft versus host disease (GVHD), and survival were all determined from clinical note documentation. CB-related complaints of toxicity were graded with the Common Terminology Criteria for Adverse Events, version 4.03, from the National Institutes of Health, which allows for the characterization and grading of toxicity. Statistical analyses, including unpaired $t$ test, log-rank test, overall survival, and CNS disease-free survival, were calculated with the Kaplan-Meier method in R (version 2.15.2) with the survival package.

\section{Results \\ Overall outcomes}

Median follow-up time for overall cohort was 19.3 months. In the CB group, median followup was 21.6 months. Three-year CNS disease-free survival and overall survival were $94.7 \%$ (95\% confidence interval [CI], 85.2-100) and 62.7\% (95\% CI, 47.9-82.0). Median survival length was 25.1 months. In the non-CB group, median follow-up was 11.6 months. Threeyear CNS disease-free survival and overall survival in this population were $81.8 \%$ (95\% CI, 61.9-100) and 51.5\% (95\% CI, 32.9-80.5). Median survival length was 19.3 months. The Kaplan-Meier survival curves displaying overall survival and CNS disease-free survival are shown in Fig 1. Table 2 displays additional posttransplant outcomes.

\section{CNS failures}

A total of 36 patients received CB along with TBI before transplant. Within this population, 1 patient had CNS failure. Notably, this patient had a history of CNS involvement. This patient died 3 years after original transplant. Nineteen patients were not treated with $\mathrm{CB}$, but received TBI before transplant. In this cohort, 2 patients without any prior CNS disease involvement had CNS failure after transplant. By contrast, in the CB cohort, all patients without prior CNS involvement remained free of disease within the CNS. 


\section{Hematological relapse}

In the CB cohort, 10 (27.8\%) patients experienced relapse in the marrow after transplant, including the 1 patient who also developed CNS failure. Survival following posttransplant relapse was poor, with 2 patients alive to date. In the non-CB cohort, 3 (15.8\%) patients experienced relapse after transplant, including the 2 patients with CNS involvement, with only 1 alive to date. In our study population, posttransplant relapse was indicative of poor prognosis and survival.

\section{Toxicity}

Late toxicity in the CB cohort included 1 instance of cataracts (2.8\%), which was not clinically severe enough to warrant an operation (Common Terminology Criteria for Adverse Events 4.03 grade 2). There were no cognitive complaints noted or radiation-related secondary malignancy. On follow-up, there were no issues reported related to memory, speech, motor, or functional capabilities of the patients.

\section{Transplant-related mortality}

The 2 most common complications that came associated with transplant-related mortality were GVHD and infection. In our total patient population, 16 confirmed posttransplant deaths were due to these 2 causes. Table 2 documents the instances of GVHD and infection that were indicated in death. Overall, GVHD was noted in 41 of the 55 individuals. The most common sites of GVHD were the skin and the gastrointestinal tract, and their severity varied greatly. The sites indicated in patients for GVHD are shown in Table 3.

\section{Discussion}

The present study suggests that $6 \mathrm{~Gy} \mathrm{CB}$ is well-tolerated in an adult population with highrisk ALL. Overall, CB appears to benefit patients at risk of CNS involvement, but without a history of CNS disease. There were no reports of severe long-term cognitive toxicity or radiation-related secondary malignancy from this low-dose prophylactic $\mathrm{CB}$. This suggests that $6 \mathrm{~Gy} \mathrm{CB}$ is a safe regimen because it is well-tolerated and efficacious.

The true CNS failure rate of ALL in adults is unknown. Although the size of the CB cohort was small, patients with past CNS involvement were more likely to have CNS relapse than those without prior CNS involvement, even though they received both CB and IT chemotherapy. This is consistent with a past study completed by Hamdi et al in adult ALL patients, suggesting that prior CNS disease indicates higher relapse rates and overall poorer prognosis after transplant. ${ }^{16}$ Notably, patients in our CB cohort with CNS failure all had prior involvement, suggesting that 6 Gy may not be adequate in this population and that these patients may require treatment intensification. Extrapolating from the pediatric ALL literature, higher dose CB may be required in this setting. Hiniker et al recently showed that $24 \mathrm{~Gy}$ CB or craniospinal irradiation in children with CNS+ ALL was well tolerated and effective. $^{17}$

The limitations of this study include the small sample size, lack of routine cognitive tests, and its retrospective nature. This study was not powered to detect survival differences. 
Additionally, the non-CB group had a higher proportion of patients receive cord blood transplants with mini-TBI as part of their conditioning regimens. These treatment differences limit the conclusions that can be drawn from this comparison. However, studies have shown that matched unrelated donor or cord blood transplants could yield similar results compared with matched related donor transplants. ${ }^{1}$ Furthermore, a higher proportion of patients in the non-CB group experienced transplant-related mortality and had relapsed disease as indication for transplant, which may also drive survival differences. Despite these limitations, this remains the largest study to date that we are aware of evaluating low-dose prophylactic 6 Gy CB in adult ALL transplants. It suggests that CB should not be abandoned in the adult population based on pediatric literature looking at higher dosages of radiation.

At our institution, we treat high-risk adult ALL patients without prior CNS involvement with low-dose 6 Gy CB prophylaxis before TBI and transplant. The exact age cutoff where CB is suitable remains unclear. Although there were 2 14-year olds undergoing CB without significant toxicity, there were too few patients to draw definitive conclusions from regarding the lower age range limit. Counseling with patients of the risks that come with $\mathrm{CB}$ such as cataracts and cognitive effects is given with an understanding that their risks are low. At our institution, $\mathrm{CB}$ is given over 3 sequential days before initiating the transplant regimen. In children, Vora et al recently showed that 12 to 24 Gy CB did not improve survival except in high-risk ALL children with past CNS involvement. ${ }^{10}$ Our general approach for pediatric ALL is that we use alternatives such as IT MTX for CNS prophylaxis. Adults with prior CNS involvement who received effective IT chemotherapy without CB have been observed to have poor prognosis. ${ }^{18}$ In this particular group, CNS treatment intensification should be considered.

\section{Conclusions}

The results of this study show that $6 \mathrm{~Gy} \mathrm{CB}$ is well-tolerated in the short and long term in the adult ALL population as a part of a radiation-based conditioning regimen. Low-dose CB may be considered in adults with high risk leukemia without prior CNS involvement to reduce the likelihood of recurrence.

\section{References}

1. Pui C-H, Evans WE. Treatment of acute lymphoblastic leukemia. N Engl J Med. 2006; 354:166178. [PubMed: 16407512]

2. Jabbour E, Thomas D, Cortes J, et al. Central nervous system prophylaxis in adults with acute lymphoblastic leukemia: Current and emerging therapies. Cancer. 2010; 116:2290-2300. [PubMed: 20209620]

3. Gökbuget N, Hoelzer D. Recent approaches in acute lymphoblastic leukemia in adults. Rev Clin Exp Hematol. 2002; 6:114-141. [PubMed: 12196212]

4. Chessells JM, Hall E, Prentice HG, et al. The impact of age on outcome in lymphoblastic leukaemia; MRC UKALL X and XA compared: A report from the MRC Paediatric and Adult Working Parties. Leukemia. 1998; 12:463-473. [PubMed: 9557602]

5. Pui CH, Relling MV, Downing JR. Acute lymphoblastic leukemia. N Engl J Med. 2004; 350:15351548. [PubMed: 15071128]

6. Omura GA, Moffitt S, Vogler WR, et al. Combination chemotherapy of adult acute lymphoblastic leukemia with randomized central nervous system prophylaxis. Blood. 1980; 55:199-204. [PubMed: 6928104] 
7. Alexander BM, Wechsler D, Braun TM, et al. Utility of cranial boost in addition to total body irradiation in the treatment of high risk acute lymphoblastic leukemia. Int J Radiat Oncol Biol Phys. 2005; 63:1191-1196. [PubMed: 15978741]

8. Ganem G, Kuentz M, Bernaudin F, et al. Central nervous system relapses after bone marrow transplantation for acute lymphoblastic leukemia in remission. Cancer. 1989; 64:1796-1804. [PubMed: 2676139]

9. Waber DP, Turek J, Catania L, et al. Neuropsychological outcomes from a randomized trial of triple intrathecal chemotherapy compared with 18 Gy cranial radiation as CNS treatment in acute lymphoblastic leukemia: Findings from Dana-Farber Cancer Institute ALL Consortium Protocol 95-01. J Clin Oncol. 2007; 25:4914-4921. [PubMed: 17971588]

10. Vora A, Andreano A, Pui CH, et al. Influence of cranial radiotherapy on outcome in children with acute lymphoblastic leukemia treated with contemporary therapy. J Clin Oncol. 2016; 34:919-926. [PubMed: 26755523]

11. Li Y, Vijayanathan V, Gulinello M, et al. Intrathecal methotrexate induces focal cognitive deficits and increases cerebrospinal fluid homocysteine. Pharmacol Biochem Behav. 2010; 95:428-433. [PubMed: 20332001]

12. Finkelstein Y, Zevin S, Raikhlin-Eisenkraft B, et al. Intrathecal methotrexate neurotoxicity: Clinical correlates and antidotal treatment. Environ Toxicol Pharmacol. 2005; 19:721-725. [PubMed: 21783548]

13. Conklin HM, Krull KR, Reddick WE, et al. Cognitive outcomes following contemporary treatment without cranial irradiation for childhood acute lymphoblastic leukemia. J Nat Cancer Inst. 2012; 104:1386-1395. [PubMed: 22927505]

14. Fukano R, Nishimura M, Ito N, et al. Efficacy of prophylactic additional cranial irradiation and intrathecal chemotherapy for the prevention of CNS relapse after allogeneic hematopoietic SCT for childhood ALL. Pediatr Transplant. 2014; 18:518-523. [PubMed: 24815062]

15. Sundaresan N, Gutierrez FA, Larsen MB. Radiation myelopathy in children. Ann Neurol. 1978; 4:47-50. [PubMed: 697325]

16. Hamdi A, Mawad R, Bassett R, et al. Central nervous system relapse in adults with acute lymphoblastic leukemia after allogeneic hematopoietic stem cell transplantation. Biol Blood Marrow Transplant. 2014; 20:1767-1771. [PubMed: 25017763]

17. Hiniker SM, Agarwal R, Modlin LA, et al. Survival and neurocognitive outcomes after cranial or craniospinal irradiation plus total-body irradiation before stem cell transplantation in pediatric leukemia patients with central nervous system involvement. Int J Radiat Oncol Biol Phys. 2014; 89:67-74. [PubMed: 24725690]

18. Surapaneni UR, Cortes JE, Thomas D, et al. Central nervous system relapse in adults with acute lymphoblastic leukemia. Cancer. 2002; 94:773-779. [PubMed: 11857312] 

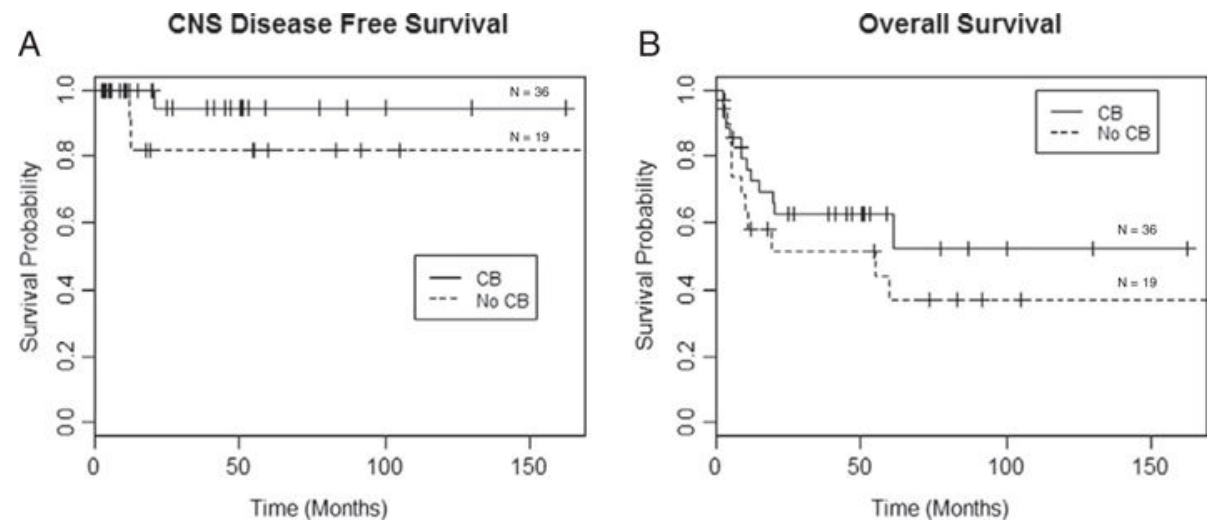

Figure 1.

Kaplan-Meier survival curves. CB group $(n=36)$; no-CB group $(n=19)$. (A) CNS diseasefree survival for patients according to whether $6 \mathrm{~Gy} \mathrm{CB}$ was given in addition to total body irradiation before transplant. The 3-year CNS disease-free survival was $94.7 \%$ (95\% CI, 85.2-100) for the CB group and 81.8\% (95\% CI, 61.9-100) for the no-CB group ( $P=.168)$. (B) Overall survival for patients according to whether $6 \mathrm{~Gy} \mathrm{CB}$ was given in addition to total body irradiation before transplant. The 3-year overall survival was $62.7 \%$ (95\% CI, 47.982.0) for the CB group and 51.5\% (95\% CI, 32.9-80.5) for the no CB group $(P=.309)$. CB, cranial boost; CI, confidence interval; CNS, central nervous system. 


\section{Table 1}

Patient characteristics

\begin{tabular}{lll}
\hline & CB & No CB \\
\hline Total number & 36 & 19 \\
Male & $22(61.1 \%)$ & $9(47.4 \%)$ \\
Female & $14(38.9 \%)$ & $10(52.6 \%)$ \\
Phenotype & B cell: $27(75 \%)$ & B cell: 11 (57.9\%) \\
& T cell: $6(16.7 \%)$ & T cell: $5(26.3 \%)$ \\
Philadelphia chromosome & Mixed phenotype ALL: 3 (8.3\%) & Mixed phenotype ALL: 3 (15.8\%) \\
Intrathecal chemotherapy before transplant & $17(47.2 \%)$ & $5(26.3 \%)$ \\
IV high-dose methotrexate & $26(72.2 \%)$ & $14(73.7 \%)$ \\
Type of transplant & $3(8.3 \%)$ & $0(0 \%)$ \\
& Sibling: $15(41.7 \%)$ & Sibling: $1(5.3 \%)$ \\
& VUD: $18(50 \%)$ & VUD: $5(26.3 \%)$ \\
Relapsed or refractory disease as indication for transplant & $14(38.9 \%)$ & Cord blood: $12(63.2 \%)$ \\
Transplant during first remission & Cord blood: $3(8.3 \%)$ & Autologous: $1(5.3 \%)$ \\
Prior CNS involvement & $22(61.1 \%)$ & $12(63.2 \%)$ \\
\hline
\end{tabular}

ALL, acute lymphoblastic leukemia; CB, cranial boost; CNS, central nervous system; IV, intravenous; VUD, voluntary unrelated donor. 
Table 2

\section{Posttransplant outcomes}

\begin{tabular}{lll}
\hline & CB & No CB \\
\hline Median follow-up & 21.6 months & 11.6 months \\
Posttransplant relapse & $10(27.8 \%)$ & $3(15.8 \%)$ \\
Posttransplant CNS failure & $1(2.8 \%)$ & $2(10.5 \%)$ \\
Median overall survival & 25.1 months & 19.3 months \\
Median survival in population with prior CNS disease & 38.7 months & 29.8 months \\
Median survival in population with relapse as transplant indication & 20.1 months & 19.3 months \\
Population still alive & $19(52.8 \%)$ & $6(31.5 \%)$ \\
Population that died from complications of transplant & GVHD 3 (8.3\%) & GVHD 5 (26.3\%) \\
& Infection 4 (11.1\%) & Infection 4 (21.1\%) \\
\hline
\end{tabular}

$\mathrm{CB}$, cranial boost; CNS, central nervous system; GVHD, graft versus host disease. 


\section{Table 3}

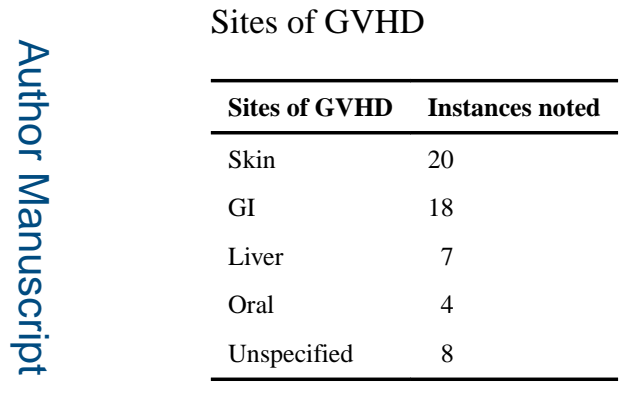

GI, gastrointestinal; GVHD, graft versus host disease. 